\title{
Prevalência e impacto da incontinência urinária na qualidade de vida de idosas institucionalizadas
}

\author{
Prevalence and impact of urinary incontinence on the quality of life of institutionalized \\ elderly women
}

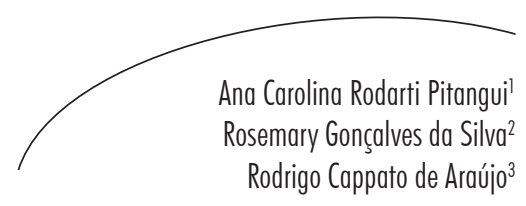

\section{Resumo}

A incontinência urinária (IU) é definida como qualquer perda involuntária de urina. Acomete mulheres de várias faixas etárias e apresenta alta incidência em idosas, afetando de forma significativa a qualidade de vida. O objetivo deste estudo foi determinar a prevalência de IU em idosas institucionalizadas e verificar sua influência na qualidade de vida. A amostra foi composta por 40 idosas institucionalizadas dos municípios de Petrolina e Juazeiro que tinham idade igual ou superior a 60 anos. Inicialmente, foram coletadas informações sociodemográficas, ginecológicas e sobre a presença de IU. Em seguida, as idosas que relataram perda de urina responderam ao questionário específico King's Health Questionnaire (KHQ). A análise de dados foi feita por meio de estatística descritiva. A presença de IU foi referida por 47,50\% das idosas. O domínio do KHQ que sofreu maior interferência na qualidade de vida foi o de percepção de saúde com 51,31\%; nos outros domínios, o impacto da IU foi considerado baixo, mas na escala de sintomas as idosas relataram que a IU afeta mais ou menos ou muito sua vida. Foi possível concluir que é elevada a prevalência de IU em idosas institucionalizadas, e observar que muitas vezes essas mulheres convivem com estes sintomas como algo intrínseco ao envelhecimento, não percebendo o quanto afetam sua qualidade de vida.

\section{Abstract}

Urinary incontinence (UI) is defined as any involuntary loss of urine. It affects women of all ages and has high incidence in elderly women, significantly affecting the quality of life. This study aimed to determine the prevalence of UI in institutionalized elderly women and verify their influence on the quality of life. The sample consisted of 40 institutionalized elderly women in the municipalities of Petrolina and Juazeiro who

\footnotetext{
Departamento de Fisioterapia. Programa de Pós-graduação em Hebiatria. Universidade de Pernambuco. Petrolina, PE, Brasil.

2 Curso de Fisioterapia. Universidade de Pernambuco. Petrolina, PE, Brasil.

Departamento de Fisioterapia, Programas de Pós-graduação em Educação Física e em Hebiatria. Universidade de Pernambuco. Petrolina, PE, Brasil.
}

Correspondência / Correspondence

Ana Carolina Rodarti Pitangui

Departamento de Fisioterapia da Universidade de Pernambuco-UPE

Campus Petrolina - BR 203 Km 2 S/N, Cidade Universitária

56330-000 Petrolina, PE, Brasil

E-mail: carolina.pitangui@upe.br

Palavras-chave:

Incontinência urinária/ epidemiologia. Qualidade de vida. Idoso/Mulheres. Instituição de Longa Permanência para Idosos. Análise de dados. Pernambuco / Brasil. 
were aged 60 or more years. Initially, sociodemographic and gynecological information, and about the presence of UI was collected. Then the elderly women who reported loss of urine answered the specific King's Health Questionnaire (KHQ). Data analysis was performed using descriptive statistics. The presence of urinary incontinence was reported by $47.50 \%$ of the elderly women. The domain of the KHQ that suffered the greatest interfering with the quality of life was the perception of health $(51.31 \%)$; in the other domains the impact of UI was considered low, however in the scale of symptoms patients reported that UI affects more or less or very much their lives. It is possible to conclude that the prevalence of UI in institutionalized elderly women is high, and to observe that often these women live with such symptoms as intrinsic to aging, not realizing how much their quality of life is affected.

\section{INTRODUÇÃO}

A incontinência urinária (IU) é definida como qualquer perda involuntária de urina, sendo considerada atualmente como um problema de saúde pública. ${ }^{1}$ Segundo a Sociedade Internacional de Continência (International Continence Society - ICS), a IU é considerada como um sintoma ou um sinal, podendo ser classificada como incontinência urinária de esforço (IUE), urge-incontinência (UI) ou incontinência urinária mista (IUM), que seria a associação das duas incontinências anteriores. ${ }^{2}$

Este sintoma acomete mulheres de várias faixas etárias, tendo maior prevalência em idosas, gestantes, pacientes com predisposição genética, obesas, mulheres com infecção urinária, pacientes neurológicos e pacientes submetidas ao parto normal, sendo responsável por causar inúmeros transtornos à qualidade de vida. ${ }^{3}$

Especificamente em relação à etiologia da IU no envelhecimento, postula-se que estaria relacionada ao próprio processo fisiológico, visto que esta é uma etapa da vida composta por maior vulnerabilidade às doenças, podendo influenciar diretamente em disfunções nas vias urinárias inferiores, contribuindo com o surgimento da IU e aumentando sua prevalência entre os idosos. ${ }^{4}$ Desta forma, a IU irá ocasionar impacto altamente negativo no desempenho desses pacientes, causando diversos transtornos físicos, econômicos, psicológicos, emocionais, sexuais e sociais, refletindo na condição de saúde e interferindo diretamente no bem-estar social e na qualidade de vida. ${ }^{5}$
Key words: Urinary Incontinence/epidemiology. Quality of life. Elderly/ Women. Establishing Long Term Elderly. Data Analysis. Pernambuco/Brazil.
A avaliação do impacto da IU na qualidade de vida do indivíduo pode ser realizada por aplicação de diversos questionários. O King's Health Questionnaire (KHQ) é uma ferramenta traduzida e validada para o português, que avalia a presença de sintomas de IU e seu impacto relativo. Permite a mensuração global e também avalia o impacto dos sintomas nos vários aspectos da individualidade na qualidade de vida. ${ }^{6}$

O emprego de medidas de mensuração da qualidade de vida é de extrema relevância para a prática clínica e científica, pois são importantes ferramentas de mensuração da efetividade de intervenções terapêuticas, podendo ser utilizadas como respaldo para sua indicação. As principais formas terapêuticas empregadas no tratamento da IU são compostas pelos tratamentos conservador e cirúrgico. ${ }^{7}$ A finalidade das modalidades conservadoras é minimizar os sintomas da IU e melhorar a qualidade de vida do indivíduo, visto que, na maioria das vezes, não há cura. ${ }^{8}$

Diversas pesquisas já evidenciam que o tratamento fisioterapêutico é de grande importância e demonstra bons resultados no manejo da IU, sendo recomendado pela Sociedade Internacional de Continência como primeira opção de escolha, por apresentar menor prevalência de reações adversas e ter como vantagens o baixo custo, além de poder em muitos casos evitar um procedimento cirúrgico. ${ }^{8,9}$

Com base no exposto, e devido à relevância para os profissionais de saúde e para as pacientes de se mensurar o impacto da IU no bem-estar social do indivíduo, o objetivo deste estudo 
foi determinar a prevalência de IU em idosas institucionalizadas e verificar sua influência na qualidade de vida.

\section{MATERIAIS E MÉTODOS}

Trata-se de estudo transversal com abordagem descritiva, desenvolvido em instituições de idosos nos municípios de Petrolina-PE e Juazeiro-BA. O período de coleta de dados foi de julho a agosto de 2011, tendo no total uma amostra por conveniência composta por 40 idosas.

Adotou-se como critério de inclusão: ter idade igual ou superior a 60 anos, compreensão da língua portuguesa, serem orientadas quanto ao tempo e espaço. Foram excluídas as voluntárias que apresentaram transtornos psiquiátricos, déficit cognitivo e incapacidade de deambulação.

Foi realizado teste piloto em dez idosas para verificar a compreensão dos instrumentos de coleta de dados e a existência de possíveis vieses. O estudo foi aprovado pelo Comitê de Ética em Pesquisa da Universidade de Pernambuco sob registro CEP/UPE 065/11, e todas as participantes assinaram o termo de consentimento livre e esclarecido, redigido conforme a Resolução no 196/96 do Conselho Nacional de Saúde.

\section{Procedimentos}

$\mathrm{Na}$ abordagem inicial das participantes, foram coletadas informações sociodemográficas (idade, etnia, situação conjugal), antecedentes ginecológicos, dados sobre a perda urinária, tratamento prévio para IU e consulta médica decorrente da IU.

Para as idosas que relatavam perda de urina, foi aplicado o questionário específico King's Health Questionnaire (KHQ), traduzido e validado para a língua portuguesa por Fonseca et al. ${ }^{10} \mathrm{O}$ KHQ é composto por 30 perguntas distribuídas em nove domínios que abordam, respectivamente: percepção da saúde, impacto da incontinência, limitações nos desempenhos das tarefas, limitação física, limitação social, relacionamento pessoal, emoções, sono/energia e medidas de gravidade.

O instrumento também possui escala de sintomas, composta pelos itens: aumento da frequência urinária, noctúria, urgência, hiperreflexia vesical, IUE, enurese noturna, incontinência no intercurso sexual, infecções urinárias e dor na bexiga.

A todas as respostas do KHQ são atribuídos valores numéricos, que são somados e avaliados por domínios. As respostas são baseadas em uma escala numérica crescente e proporcional à intensidade da queixa ( $0=$ não/não se aplica; $1=$ um pouco/às vezes; $2=$ mais ou menos/ várias vezes; $3=$ muito/sempre), com exceção ao domínio "percepção geral de saúde", que tem cinco opções de resposta: muito boa, boa, regular, ruim e muito ruim. A pontuação é feita por cada um dos seus domínios, não havendo escore geral. Os escores variam de 0 a 100, e quanto maior a pontuação, pior é a qualidade de vida relacionada ao domínio.

A aplicação do KHQ foi realizada por meio de entrevista face a face, conduzida por avaliador devidamente treinado.

\section{Análise Estatística}

A análise de dados foi realizada por meio da construção de planilha eletrônica no software aplicativo Microsoft Excel. Após codificação das variáveis em dicionário de dados e da validação do banco de dados por dupla entrada, realizou-se a análise exploratória das variáveis. Os dados foram apresentados por meio de estatística descritiva. As variáveis contínuas foram apresentadas sob a forma de medidas de tendência central e dispersão, enquanto categóricas, sob a forma de frequências absolutas e relativas. A análise estatística foi realizada no programa Statistical Package for Social Sciences (SPSS), versão 16.0 para Windows. 


\section{RESULTADOS}

Das 40 idosas participantes da pesquisa, $19(47,50 \%)$ apresentavam IU. Nos dados sociodemográficos, constatou-se que a idade média das participantes foi de 74,97 $\pm 8,54$ anos, sendo que a idade mínima foi de 60 anos e a máxima, 98 anos.

Das idosas da amostra, identificou-se que 38 (95\%) tinham fundamental incompleto e eram oriundas da zona rural. Quanto à etnia, $17(42,50 \%)$ eram brancas, 13 (32,50\%) eram negras, nove $(22,50 \%)$ eram pardas e apenas uma $(2,50 \%)$ era amarela.

Em relação ao estado civil, verificou-se que quatro $(10 \%)$ eram solteiras, seis $(15 \%)$ casadas, oito $(20 \%)$ separadas e $22(55 \%)$ viúvas. $\mathrm{Na}$ variável realização de cirurgias ginecológicas anteriores, $20(50 \%)$ idosas mencionaram ter feito, sendo a histerectomia e perineoplastia os procedimentos relatados com maior predominância, mencionados igualmente por sete $(20 \%)$ das entrevistadas.

O uso de medicamentos foi relatado por todas as idosas (100\%), observando-se o emprego de medicamentos associados, sendo os antihipertensivos relatados com maior incidência, por $60 \%(\mathrm{n}=24)$ das idosas.

Sobre o impacto da IU na qualidade de vida das pacientes, pode-se verificar, na tabela 1 , as médias e desvios-padrão das pontuações dos domínios avaliados pelo KHQ referentes a percepção da saúde, impacto da IU, limitação nas atividades de vida diárias (AVDs), limitações físicas, limitações sociais, emoções, sono/energia e medidas de gravidade.

O único domínio não contemplado foi o de Relações pessoais, pois a resposta "não foi aplicável" foi referida por 17 entrevistadas e outras duas relataram que a IU não interferia nas suas relações pessoais.

Tabela 1 - Pontuação da qualidade de vida das idosas segundo os domínios do King's Health Questionnaire (KHQ). 2011.

\begin{tabular}{cc}
\hline Domínios do KHQ & Média \pm desvio padrão \\
\hline Percepção da saúde & $51,31 \pm 22,78$ \\
Impacto da IU & $26,31 \pm 28,50$ \\
Limitações das AVDs & $7,89 \pm 12,87$ \\
Limitações físicas & $11,40 \pm 17,61$ \\
Limitações sociais & $6,14 \pm 11,39$ \\
Relações pessoais & - \\
Emoções & $16,37 \pm 23,23$ \\
Sono/energia & $20,17 \pm 30,21$ \\
Medidas de gravidades & $29,82 \pm 25,80$ \\
\hline
\end{tabular}


Além disso, também foram avaliados pela escala do KHQ quais sintomas urinários mais afetavam as idosas incontinentes. $\mathrm{Na}$ tabela 2, pode-se observar a prevalência e a quantificação dos sintomas segundo as participantes do estudo.

Tabela 2 - Escala dos sintomas urinários relatados pelas idosas incontinentes do estudo. 2011.

\begin{tabular}{l|c|c|c|c}
\hline \multicolumn{5}{c}{ Quais são seus problemas de bexiga e quanto eles afetam você? } \\
\hline \multirow{2}{*}{ Sintomas } & Nenhum & Um pouco & $\begin{array}{c}\text { Mais ou } \\
\text { menos }\end{array}$ & Muito \\
\cline { 2 - 5 } & $\mathrm{n}(\%)$ & $\mathrm{n}(\%)$ & $\mathrm{n}(\%)$ & $\mathrm{n}(\%)$ \\
\hline Aumento da frequência urinária & - & $6(31,58)$ & $4(21,05)$ & $9(47,37 \%)$ \\
Noctúria & $2(10,53)$ & $4(21,05)$ & $3(15,79)$ & $10(52,63 \%)$ \\
Urgência miccional & - & $6(31,58)$ & $4(21,05)$ & $9(47,37 \%)$ \\
Urge incontinência & - & $12(63,16)$ & $4(21,05)$ & $3(15,79 \%)$ \\
IU de esforço & $5(26,32)$ & $6(31,58)$ & $4(21,05)$ & $4(21,05 \%)$ \\
Enurese noturna & $10(52,63)$ & $5(26,32)$ & $2(10,53)$ & $2(10,53 \%)$ \\
Incontinência durante a relação sexual & $19(100)$ & - & - & - \\
Infecções urinárias frequentes & $7(36,84)$ & $3(15,79)$ & $8(42,11)$ & $1(5,26 \%)$ \\
Dor na bexiga & $8(42,11)$ & $5(26,32)$ & $6(31,58)$ & - \\
Outro problema na bexiga & $12(63,16)$ & $4(21,05)$ & $3(15,79)$ & - \\
\hline
\end{tabular}

\section{DISCUSSÃO}

O envelhecimento é um processo natural que se dá durante o curso da vida do indivíduo e submete o corpo humano a inúmeras mudanças físicas e funcionais, com repercussões nas condições de saúde física e psicológica. Muitas dessas mudanças são progressivas, e ocasionam diminuição na capacidade funcional dos indivíduos. ${ }^{11}$

Pesquisas apontam que o envelhecimento da população ocorre de maneira acelerada, e que até o ano de 2025 os idosos constituirão 14\% da população mundial. ${ }^{12}$ Desta forma, o objetivo deste estudo foi determinar a prevalência e o impacto da IU na qualidade de vida de idosas institucionalizadas.

A média de idade das participantes do presente estudo foi de 74,97 anos, sendo referido pela maioria o estado civil de viúvas, correspondendo a 55\% das idosas, tendo apenas $10 \%$ mencionado ser solteiras. Este percentual diverge de outros trabalhos realizados em instituições de longa 
permanência, no qual mais de $50 \%$ das idosas residentes eram solteiras. ${ }^{13,14}$

Em relação ao uso de medicamentos relatado pelas participantes, constatou-se que foi de $100 \%$, valor superior ao encontrado por outros autores, que observaram percentual de $72,1 \% 0^{15} \mathrm{e}$ $86,4^{1} 0^{14} \mathrm{em}$ idosos institucionalizados. Dentre os medicamentos empregados com maior frequência pelas idosas deste estudo, verificou-se que a maioria fazia uso de medicamentos associados, sendo predominante o uso de anti-hipertensivos.

O excesso e o uso constante de fármacos utilizados pelas idosas demonstram que tal prática é frequente nesta população e deve ser abordada com cautela. O organismo do idoso possui características específicas, em decorrência da redução da massa muscular, da água corporal e do metabolismo hepático, podendo apresentar reações adversas, tais como dificuldade de metabolização e eliminação de drogas, que podem ocasionar acúmulo de substâncias tóxicas no organismo. ${ }^{16}$

Estudos indicam, ainda, que a medicação pode ser vista como fator de risco para o aparecimento da IU, aumentando a frequência e a urgência urinária, ${ }^{17}$ pois inúmeros fármacos anti-hipertensivos afetam o funcionamento do trato urinário inferior, podendo gerar a IU..$^{18}$

Os procedimentos cirúrgicos ginecológicos aos quais as idosas foram submetidas com maior frequência foram histerectomia e perineoplastia. Segundo alguns autores, ${ }^{19-21}$ mulheres submetidas a histerectomia têm grandes chances de apresentar IU. A retirada do útero compromete o bom funcionamento da musculatura de assoalho pélvico, podendo causar danos na sustentação da bexiga e da uretra, e ser responsável pelo aumento da prevalência da IU. ${ }^{20,21}$ Além disso, com o avanço da idade, ocorre atrofia da uretra, com enfraquecimento da musculatura do assoalho pélvico associado a perda de elasticidade uretral, que irão favorecer o aumento da frequência miccional e o surgimento da UI e da IUE. ${ }^{22}$

A prevalência de IU neste estudo foi de $47,50 \%$, percentual inferior ao encontrado em outros trabalhos, como o de Aggazzotti et al., ${ }^{23}$ que encontraram 59,8\%, e Hochi et al., ${ }^{24}$ que encontraram $67,9 \%$. Contudo, a literatura acerca da prevalência da IU ainda é incerta, sendo encontrada variação nestes valores, devido principalmente às metodologias empregadas e divergências nas amostras. ${ }^{25}$

Quanto ao impacto da IU na qualidade de vida das idosas, observaram-se baixos escores nos domínios do King's Health Questionnaire (KHQ). Acredita-se que os valores encontrados na pesquisa se dão pelo baixo grau de escolaridade das participantes, que na sua maioria relataram que os problemas de bexiga afetavam com pouca ou nenhuma intensidade sua vida.

Diversos autores também encontraram achados que corroboram o presente estudo, justificando que baixos valores nos escores do KHQ podem decorrer do fato de as pacientes se sentirem envergonhadas em responder a respeito do assunto ou por não conhecerem informações relacionadas ao tratamento da IU. ${ }^{26,27}$

No estudo atual, 95\% das idosas possuíam ensino fundamental incompleto e eram oriundas da zona rural, tendo a compreensão da IU como um processo natural do envelhecimento e não como uma patologia a ser tratada. Observou-se, ainda, a não-contemplação do domínio "relações pessoais" do KHQ, cujas perguntas se referiam à interferência do problema de bexiga nas seguintes situações: vida sexual, vida com o seu parceiro e vida com seus familiares. Acredita-se que isso se deu porque a maioria das participantes eram viúvas e pela elevada média de idade.

A não-contemplação deste item também foi confirmada pelos resultados encontrados na aplicação da escala de sintomas urinários, na qual se verificou que $100 \%$ das participantes responderam que a IU não afetava sua vida sexual. Desta forma, é possível que este resultado se deva ao fato de as participantes não apresentarem vida sexual ativa, e não em decorrência de haver impacto negativo na qualidade de vida neste domínio.

Quanto à interferência do aumento da frequência miccional da IUE e da UI na vida 
das idosas, respectivamente, $68,42 \%, 42,10 \%$ e $38,84 \%$ responderam aos itens "mais ou menos" e "muito", percentuais considerados significativos. Contudo, cabe dar destaque ao domínio do questionário KHQ "percepção geral da saúde", que obteve média de $51,31 \%$, valor superior a outros estudos, que encontraram escore médio de $45,2 \% .{ }^{28}$ Assim, as pontuações encontradas neste domínio e na escala de sintomas contradizem o relatado pelas idosas no domínio "impacto da IU”, que obteve escore baixo. Esses dados confirmam que a IU interfere na qualidade de vida das idosas, mas que o grupo de mulheres analisadas convive com este sintoma como algo intrínseco ao processo de envelhecimento, não percebendo seu impacto na qualidade de vida.

Outro item que corrobora esta conclusão foi o valor de noctúria, que apresentou percentual significativo de 52,63\%, sendo relatado pelas idosas como algo que afeta muito sua qualidade de vida.

Infelizmente, podem-se constatar algumas limitações neste estudo, como o tamanho amostral; no entanto, a quantidade de idosos institucionalizados que atendiam aos critérios de inclusão e exclusão deste estudo nos municípios estudados era pequena. Sugere-se que sejam realizados novos estudos que avaliem não apenas o impacto da IU na qualidade de vida das idosas, mas também a eficácia de tratamentos conservadores. Além disso, a amostra deste estudo não pode servir de referência para a realidade nacional, sendo recomendado o desenvolvimento de futuras pesquisas que comparem a realidade de classes sociais e regiões diferentes.

Com base no exposto, acredita-se que os dados coletados neste estudo serão de grande importância para futuras intervenções em outros serviços de fisioterapia e nos já existentes nessas instituições, que se preocupam apenas com patologias ortopédicas e neurológicas, e não abordam a área de uroginecologia.

Neste sentido, observa-se a necessidade de implantação de novas práticas de atuação que visem aprevençãoetratamento daIU, melhorando a qualidade de vida das idosas institucionalizadas e minimizando os inconvenientes causados por essa sintomatologia.

\section{CONCLUSÃO}

Com base nos resultados deste estudo, foi possível concluir que é elevada a prevalência de Incontinência Urinária (IU) em idosas institucionalizadas. E apesar da maioria dos domínios do questionário KHQ não apresentar valores significativos, verificou-se, através do domínio "percepção geral" e pela escala de sintomas, que a IU afeta consideravelmente a qualidade de vida desta população, que normalmente, por desconhecimento, acaba convivendo com esta queixa como algo inerente ao envelhecimento.

Desta forma, é cabível adotar medidas de intervenção e orientação para minimizar ou eliminar os inconvenientes gerados pela IU, proporcionando assim qualidade de vida a este público.

\section{REFERÊNCIAS}

1. Abrams P, Cardozo L, Fall M,Griffithsn D, Rosier P, Ulmsten $\mathrm{U}$, et al. The standardization of terminology of lower urinary tractfunction: repot from the standardization sub-committee of the international continence society. Urology. 2003;61(1):37-49.

2. Patrick DL, Martin ML, Bushnell DM, Marquis P, Andrejasich CM, Buesching DP. Cultural adaptation of a quality-of-life measure for urinary incontinence. Eur Urol 1999;36:427-35.
3. Huang AJ, et al. Quality-of-life impact and treatment of urinary incontinence in ethnically diverse old women. Arch Intern Med 2006;166:2000-6.

4. Fantl JA. The lower urinary tract in women-effect of aging and menopause on continence. Exp Gerontol 1994;29(3-4):417-22.

5. Van der Vaart CH,De Leuw JR, Roovers JP, et al. The effect of urinary incontinence and overactive bladder symptoms on quality of life in young women. BJU Int 2002;90:544-9. 
6. Kelleher C. Quality of life and urinary incontinence. Baillieres Best Pract Res Clin Obstet Gynaecol 2000;14(2):363-79.

7. Amaro JA, Gameiro MO, Moreira EH. Exercícios perineais. In: Ribeiro RM, Rossi P, Pinotti JA, editores. Uroginecologia e cirurgia vaginal. São Paulo: Roca; 2001. p. 5-62.

8. Dancona CAL, Júnior NRN. Aplicações clínicas da urodinâmica. 3.ed. São Paulo: Atheneu; 2001

9. Neumann PB, Grimmer KA, Grant RE, Gill VA. Physiotherapy for female stress urinary incontinence: a multicentre observational study. Aust N Z J Obstet Gynaecol 2005;45:226-32.

10. Fonseca ESM, Camargo ALM, Castro RA, Sartori MGF, Fonseca MCM, lima GR, et al. Validação do questionário de qualidade de vida (King's Health Questionaire) em mulheres brasileiras com incontinência urinária. Rev Bras Ginecol Obstet 2005;27(5):235-42.

11. Gozoni ML, Passarelli MCG. Farmacologia e Terapêutica. In: Freitas EV de, Py L. Tratado de Geriatria e Gerontologia. $3^{a}$ ed. Rio de Janeiro: Guanabara Koogan, 2011. p.1046-53.

12. Coelho Filho JM, Ramos LR. Epidemiologia do envelhecimento no Nordeste do Brasil: resultados e inquérito domiciliar. Rev Saude Publica 1999;33(5):445-53.

13. Moraes IAL. A saúde do idoso no contexto asilar: Implicações da institucionalização e as ações interventivas de enfermagem [dissertação]. Rio de Janeiro: Faculdade de Enfermagem, Universidade do Estado do Rio de Janeiro; 2002.

14. Lazari ICF, Marota AG, Lojudice DC. Avaliação da qualidade de vida de idosas comincontinência urinária: idosas institucionalizadas em uma instituição de longa permanência. Rev. Bras. Geriatr. Gerontol 2009;12(1):103-12.

15. Loyola Filho AI, Uchoa E, Guerra HL, Firmo JOA, Costa- Lima MF. Prevalência e fatores associados à automedicação: resultados do projeto Bambuí. Rev Saúde Pública 2002;36(1):55-62.

16. Galato D, Silva ES da, Tiburcio L de S. Estudo de utilização de medicamentos em idosos residentes em uma cidade do Sul de Santa Catarina (Brasil): um olhar sobre a polimedicação. Ciênc. saúde coletiva 2010;15(6):2899-905.
17. Berlezi EM, Fiorin AAM, Bilibio PVF, Kirchner RM, Oliveira KR. Estudo da incontinência urinária em mulheres climatéricas usuárias e não usuárias de medicação anti-hipertensiva. Rev. Bras. Geriatr. Gerontol 2011;14(3):415-23.

18. Higa R, Lopes MHBM, Reis MJ. Fatores de risco para incontinência urinária na mulher. Rev Esc Enferm 2008;42(1):187-92.

19. Milsom I, Ekelund P, Molander U, Arvidsson L, Areskoug B. The influence of age, parity, oral contraception, hysterectomy and menopause on the prevalence of urinary incontinence in women. J Urol. 1993;149(6):1459-62.

20. Brown JS, Seeley DG, et al. Urinary incontinence in older women: who is at risk? Study of Osteoporotic Fractures Research Group. Obstet Gyneco. 1996;87(5 Pt 1):715-21.

21. Moller LA, Lose G, Jorgensen T. Risk factors for lower urinary tract symptoms in women 40 to 60 years of age. Obstet Gynecol 2000;96(3):446-51.

22. Souza RS. Anatomia do envelhecimento. In: Carvalho Filho ET, Papaleo Netto M. Geriatria: Fundamentos, Clínica e Terapêutica. 2. ed. São Paulo: Atheneu, 2002. p. 35-42.

23. Aggazzotti G, Pesce F, Grassi D, Fantuzzi G, Righi E, De Vita D, et al. Prevalence of urinary incontinence among institutionalized patients: a cross-sectional epidemiologic study in a midsized city in northern Italy. Urology 2000;56(2):245-9.

24. Hochi T, Nakahara T, Hashimoto S, et al. Prevalence of urinary incontinence among institutionalized persons aged 60 and over in Japan. Nippon Koshu Eisei Zasshi 1995;42(7):482-90

25. Ko Y, Salmon J, Lin S, Bron M. The impact of urinary incontinence on quality of life of the elderly. Am J Manag Care 2005;11(4):103-11.

26. Silva L, Lopes MHBM. Incontinência urinária em mulheres: razões da não procura por tratamento. Rev. Esc. Enferm. USP 2009;43(1):72-8.

27. Sebben V, Filho HT. Incidência da incontinência urinária em participantes do Creati do município de Passo Fundo/RS. RBCEH 2008;5(2):101-9.

28. Tamanini JTN, D'Ancona CAL, Bortega NJ, Junior Netto R. Validação do "King's Health Questionnaire" para o português em mulheres com incontinência urinária. Rev Saúde Pública 2003;37(2):203-11. 